\title{
LINEAR RESPONSE IN DYNAMICAL SYSTEMS: OPTIMISATION AND FINITE-TIME COHERENT SETS
}

\author{
FADI ANTOWN
}

(Received 14 August 2020; first published online 9 October 2020)

2020 Mathematics subject classification: primary 37H99; secondary 37C05.

Keywords and phrases: coherent set, dynamical system, linear response, Markov chain.

The linear response of a dynamical system refers to changes to properties of the system under small external perturbations. We consider two applications of linear response theory to dynamical systems. In the first application (covering two settings) we consider the optimal perturbation that (i) maximises the linear response of the equilibrium distribution of the system, (ii) maximises the linear response of the expectation of a specified observable and (iii) maximises the linear response of the rate of convergence of the system to the equilibrium distribution. We also consider problems (i) and (ii) in the time-dependent situation where the governing dynamics is not stationary. We initially solve these problems for finite-state Markov chains. We numerically apply the theory developed in the finite-state setting to stochastically perturbed dynamical systems, where the Markov chain is replaced by a matrix representation of an approximate annealed transfer operator for the random dynamical system. This research has been published in [1]. In the second setting, we consider problems (ii) and (iii) for Hilbert-Schmidt integral operators with stochastic kernels. By representing a deterministic dynamical system with additive noise as an integral operator, we develop theory to compute optimal map perturbations that address problems (ii) and (iii); we also provide numerical examples in this setting.

The second application of linear response is to finite-time coherent sets. Finite-time coherent sets represent minimally mixing objects in general nonlinear dynamics and are spatially mobile features that are the most predictable in the medium term. Under a small parameter change to the dynamical system, one can ask about the rate of change of the location and shape of the coherent sets, and one can also ask about the mixing properties (how much more or less mixing) with respect to the parameter change. We answer these questions by developing linear response theory for the eigenfunctions of

Thesis submitted to the University of New South Wales in January 2020; degree approved on 21 May 2020; supervisor Gary Froyland.

(C) 2020 Australian Mathematical Publishing Association Inc. 
the dynamic Laplace operator, from which one readily obtains the linear response of the corresponding coherent sets. We construct efficient numerical methods based on a recent finite-element approach and provide numerical examples.

\section{Reference}

[1] F. Antown, D. Dragičević and G. Froyland, 'Optimal linear responses for Markov chains and stochastically perturbed dynamical systems', J. Stat. Phys. 170 (2018), 1051-1087.

FADI ANTOWN, School of Mathematics and Statistics,

University of New South Wales, Kensington, NSW 2052, Australia

e-mail: antown.f@gmail.com 